\title{
IoT-based e-Health Framework for COVID-19 Patients Monitoring
}

\author{
Fahad Albogamy \\ Computer Sciences Program, Turabah University College \\ Taif University, P.O. Box 11099, Taif 21944, Saudi Arabia
}

\begin{abstract}
The COVID-19 pandemic, produced by the SARS$\mathrm{CoV}-2$ virus, has caused global public health emergency, with the rapid evolution and tragic consequences. The fight against this disease, whose epidemiological, clinical, and prognostic characteristics are still being studied in recent works which is forcing a change in the form of care, to include transforming some face-to-face consultations into non-face-to-face. Recently, various initiatives have emerged to incorporate the Internet of Things (IoT) in different sectors specially the health sector generally and in e-Health systems specifically. Millions of devices are connected and generating massive amounts of data. In this sense, based on the experience in the health sector in the management of the pandemic caused by COVID-19, it has been determined that monitoring potential patients of COVID-19 is still a great challenge for the latest technologies. In this paper, an IoT-based monitoring framework is proposed to help the health caregivers to obtain useful information during the current pandemic of COVID-19, thus bringing direct benefits of monitoring patient's health and speed of hospital care and cost reduction. An analysis of the proposed framework was carried out and a prototype system was developed and evaluated. Moreover, we evaluated the efficacy of the proposed framework to detect potentially serious cases of COVID-19 among patients treated in home isolation.
\end{abstract}

\section{Keywords-COVID-19; IoT; healthcare; e-Health}

\section{INTRODUCTION}

Currently, the Internet of Things (IoT) is one of the technologies that have been widely disseminated in different application contexts [1]. IoT can be defined as a technology that consists of millions of devices that are connected to the Internet [2]. This network of devices exchange, add and process information about their physical environment to provide value-added services to the end users. Thanks to the advantages provided by IoT, its use has been made possible in different sectors such as industry, tourism, health, and the environment, which has made possible the construction and formation of smart cities [3].

There has been a remarkable growth in the number of connected devices in recent years. Since 2008, these have already surpassed the number of inhabitants on earth, and could reach 75 billion connected devices by 2025 [4]. An example of this scenario is the smart lamps, which even when they are not emitting light must remain connected, waiting for a switch on, whether from a human user or from another computer system. Another example is wearable devices [5], which constantly monitor a person's physical activity, and can issue alerts if any of the indices fall outside the desirable range
[6]. With this view, the trend is that it is increasingly necessary for isolated items to connect to share information. Therefore, just as it is now uncommon to have a computer disconnected, it will soon be unusual to have an air conditioner or a coffee maker in this same situation.

Worldwide, the commercial automation and home automation areas are, among the IoT technologies, those that currently attract the most investment and with the largest installed IoT parks in the world [7]. Despite this, many other areas can also take advantage of the growth, evolution, and cost-effectiveness of this technology to expand its possibilities. Health care is one of these areas, where the use of IoT can help in various fields, whether streamlining medical care, emergency response time, predicting the occurrence of serious events, among other possibilities [8]. In all cases, the expected benefit is the improvement in the quality and life expectancy of the public using IoT technology [9].

Coronavirus is a new disease that comes from the group of coronaviruses which has seven distinct types in this family. four types of this family are mild, like a catarrh. COVID-19 was primary identified in Wuhan City, China, and has been officially named "SARS-CoV2." It causes cough, fever and difficulty of breath. The incubation period is officially two to fourteen days after exposure. Infection can range from very mild to advanced pneumonia, but it appears that $80 \%$ of cases are mild [10].

With the COVID-19 pandemic, the researches on personal health sensing equipment became even more intense [11]. Thermometers and pulse oximeters, for example, have become even more popular. One of the impacts of this was that many wearable devices, such as smart watches, incorporated these features into their latest models. using these devices, it is possible to monitor interesting data about the user's health, such as heart rate, blood oxygenation and body temperature depending on the device model [12].

The demands of the health area that can be supported by IoT are extensive, ranging from the needs in hospitals and health care establishments, through ambulances and emergency environments to home care environments. Even with all these possibilities, the healthcare area still does not make full use of IoT technologies [13].

In intensive care unit environments, for example, patients are routinely monitored by hospital equipment that usually displays data on parametric monitors, located within sight of healthcare professionals. Its main function, arguably, is to 
display the values obtained in real time, as well as to provide audible and visual alerts when values leave the normal range. In general, the generated historical data is stored in the equipment for a few hours, just so that it is possible to observe the average, maximum and minimum values for the period. However, the definitive record is made by the nursing team: periodically a professional must go near the monitor to write down the information presented in the patient's medical record [14].

As for ambulances, operating protocols may vary according to the management and purpose of the displacement. A common case of use of ambulances is when a patient is transferred in a state of urgency or emergency to a specialized hospital. When an emergency call is received requesting, for example, an ambulance to go to the home of a patient in home care, the ambulance environment (equipment and professionals) is quickly prepared based on the health status information that was provided when the call was made. If the patient (before the arrival of the ambulance) experiences any sudden change of state (for example, cardiac arrest), it will be necessary for the ambulance team to be notified by telephone, while still on the outward journey. In a perfect setting [15], during the COVID-19 pandemic, it became necessary to simultaneously monitor millions of infected people, to monitor their symptoms and carry out, if necessary and at the right time, their hospitalization[16]. In this scenario, there is a great benefit in using IoT devices to collect and analyze patient health data in real time, which is the possibility of predicting the capacity of sectors and scheduling internal patient transfers. Not only that, but there could also be an optimization in the process of external transfers, as the team could, through realtime patient data, choose the ideal moment to perform each transfer.

These factors indicate that the current way of using health sensing data can be improved, with the objective of generating benefits for both the patient and for the professionals and health services involved. These benefits are related to the agility in identifying health problems, measurable by sensors, the quick notification of health caregivers and the possibility of obtaining prognoses about the patient's health.

The contribution of this paper is to propose an IoT-based monitoring framework that can help the health caregivers to obtain useful information during the current pandemic of COVID-19. Moreover, a prototype system is developed and evaluated.

The rest of this paper is organized as follows: Section II discusses related works. Section III describes the proposed IoT-based e-health framework. Section IV elaborates the prototype simulated implementation. Section $\mathrm{V}$ discusses the experiment. In Section VI, we discuss our findings. A conclusion if this work in conducted in Section VII. Finally, Future work is proposed in Section VIII.

\section{RELATED WORK}

This section presents a set of related works that were considered for the development of this work. In [17], a review is made of the main potentialities of IoT as a mechanism to mitigate the impact of the pandemic caused by COVID-19.
Likewise, a set of applications that have been developed in different countries for the identification and control of patients with COVID-19 are presented, several of which have been articulated to the health system. In the same way, the authors highlight the possibilities of IoT to make remote and autonomous monitoring of variables such as heart rate, blood pressure and blood glucose by patients. In the same way, as challenges of this type of technology, the security of the data and the interoperability of the different devices stand out.

In [18], the authors propose an IoT system for heart rate monitoring in usability tests. The system proposed by the authors obtains the heart rate and heart rate variation data from a Bluetooth belt and sends them to a desktop application, which receives the data through a Bluetooth adapter and stores them in a database at the time that displays them graphically as a function of time. From the data captured, the system determines the level of mental stress of the user at different moments of the test.

In [19], a system for the detection of heart conditions and the identification of mental stress is proposed using the free Arduino hardware platform and a heart rate sensor compatible with it. From the data captured by the sensor, the system allows the graph of the heart rate to be displayed in real time as a function of time, as well as the possible level at which the obtained heart rate is classified (bradycardia, normal, tachycardia). The system presented by the authors does not allow the storage of the data and therefore neither the analysis of the history of the captured data.

In [20], the authors present an IoT system for the selfdiagnosis of heart diseases using a probabilistic method for the study of cardiac dynamics. The proposed system is framed in the service-oriented architecture and consists of a bluetooth LE heart rate sensor, which captures the data and sends it in real time to a mobile application, which is responsible for visualizing the data and calculating the probability. Heart rate takes a previously defined critical value. Although the proposed system includes the capture, storage and analysis layers, it does not present the behavior of the heart rate in real time at a graphical level, nor does it take into consideration the level of oxygen saturation as a study variable.

Among other applications of IoT for health care, the proposal of [21] stands out who propose a monitoring system for chronic obstructive pulmonary disease-COPD through constant measurement of body temperature, oxygen saturation and heart rate with inexpensive sensors to issue early prevention alerts. For their part, [22] present a literature review oriented to internet of things applications for home health care using IoT technology, known as IoT Health.

All the works presented in this section show IoT Health as a promising alternative for the health industry by allowing the personalization of the health service with lower labor and operational costs and facilitating the early warning of health alterations in people.

\section{PROPOSED FRAMEWORK}

In this section, a detailed description for the proposed framework is given. A generic diagram of the framework is shown in Fig. 1. It aims to provide support for healthcare 
specialist decision-maker. Additionally, it works as a component capable of generating useful information about patients through IoT technology. The proposed framework can receive data collected from sensors, process and generate relevant information and notifications in a timely manner. The proposed framework was designed for COVID-19 patients.

\section{A. IoT Data Collection Module}

An important part of data analysis is collecting patient data. IoT data collection module considered as the bridge between wearable sensors and the proposed framework. This module is built based on the IoT infrastructure for health applications. In this experiment, the Mysignals platform is used. MySignals is a hardware development platform built for e-health system [23]. Fig. 2 shows MySignals device and its different types of connected IoT sensors. The information collected by MySignals is useful for the analysis of COVID-19 patients.

The following information can be collected through MySignals:

- Body position - important to know which side the patient is on or has been in the same position for many hours (warning to prevent pressure ulcers from forming). In the case of patients with COVID-19, this data may indicate whether the patient is walking or lying down, in addition to indicating the patient's level of discomfort and stress.

- Body temperature: the body temperature data is collected more often for the caregivers. This data can also be used by crossing data from the external environment, since temperature and other external factors can also affect the patient.

- Electromyography: This sensor detects abnormal muscle electrical activity. Although not directly related to the symptoms of COVID-19, the caregivers can use it as a crossover to indicate other comorbidities that may weaken the patient in general.

- Airflow: to check the number of breaths and identify the patient's discomforts for breathing.

- Galvanic skin response: can be used to check patient relaxation and stress levels.
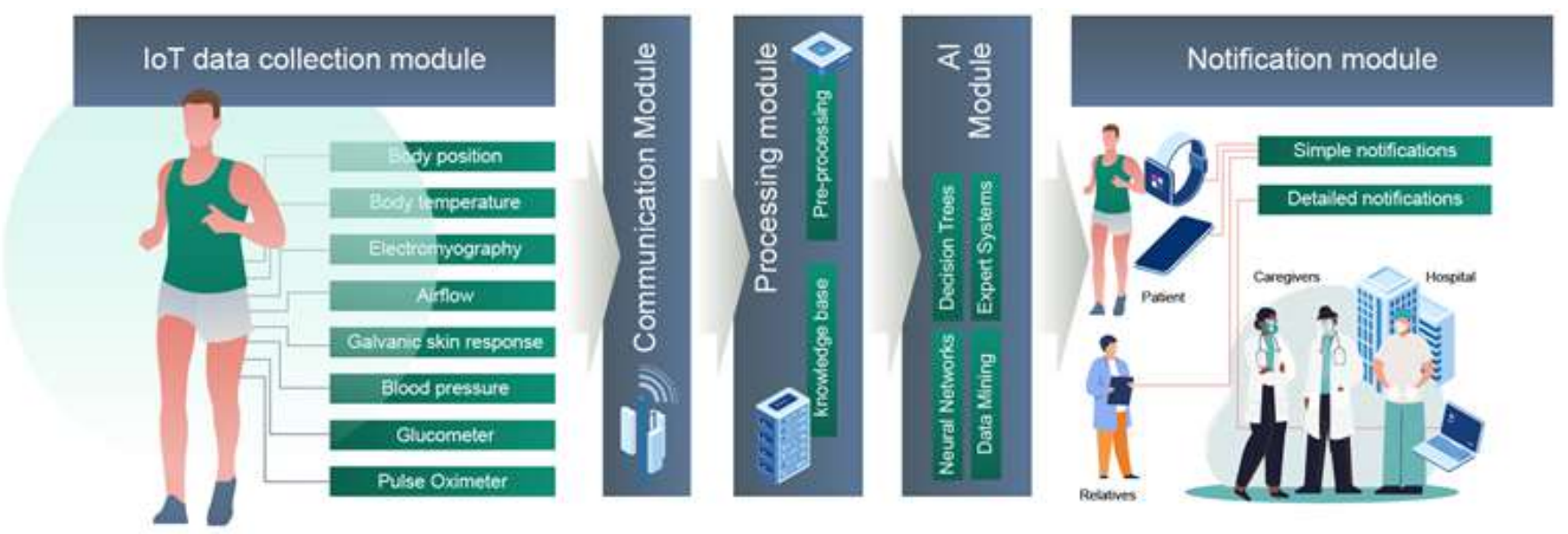

Fig. 1. Conceptional Diagram of the Proposed Framework. 


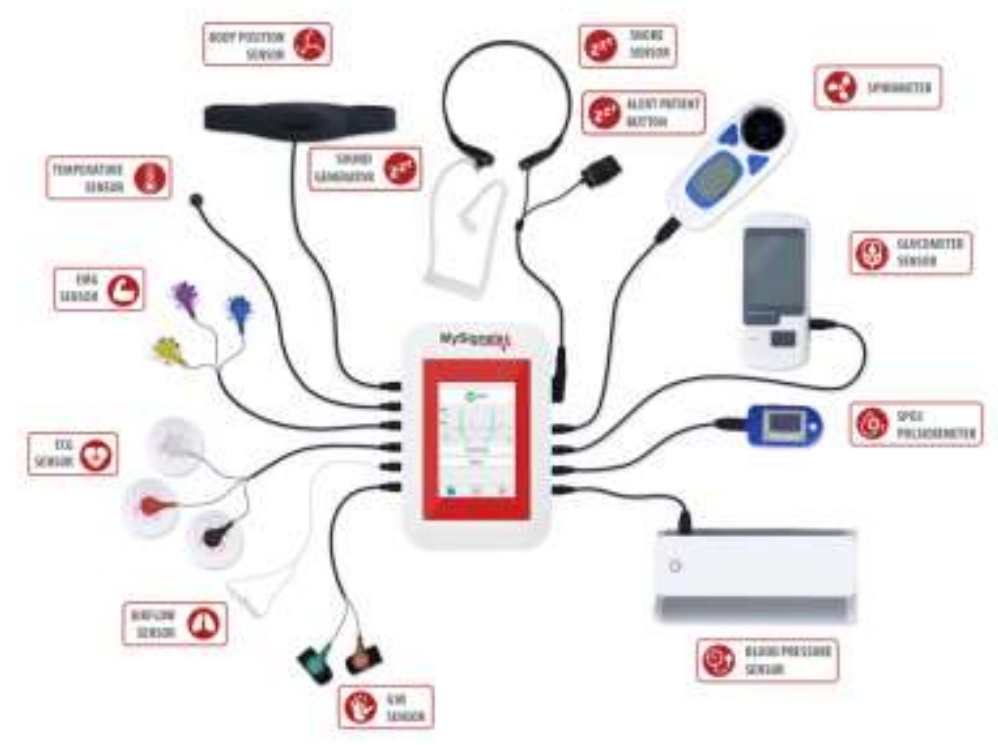

Fig. 2. MySignals Device and its different IoT Connected Sensors [23].

There are many algorithms that use AI techniques, which help in the automatic grouping and classification of data. As an example of these AI techniques, it is possible to mention:

- Neural Networks: are specific Data Mining techniques that aim to learn through examples and try to apply the same rules learned to new unclassified data.

- Data Mining: are techniques to explore data in search of patterns. The goal is to discover hidden patterns, such as time sequences or association rules between data, to classify new data.

- Decision Trees: is a technique for creating a sequence of rules that determine results through a series of logical tests.

- Expert Systems: are software that simulates a professional's decisions expert in the area, through a combination of rules or heuristics that analyze information, just like a human being would.

\section{E. Notification Module}

The notification module represents an important part of the proposed framework. In this module, each patient will receive notifications in two distinct categories: simple notifications and detailed notifications. These notifications are used by the caregivers, and can be displayed on a mobile application, monitoring screen or SMS messages sent to the patient relatives for immediate action. As mentioned before, two categories of notifications can be issued by the notification module. These categories are as follows:

- Simple notifications: This category is related to events that occur by direct analysis of data received from a sensor, by searching the knowledge base. For example, a notification could be triggered by a heart rate outside the pre-established normality or a low percentage of oxygen when analyzing the glucometer. These events trigger alerts quickly.
- Detailed notifications: This category is that of notifications triggered after analyzing the data received from various sensors, using data crossing and analysis of historical evolution. The information obtained is checked against the knowledge base to generate definitive results. For example, a patient could be categorized with their degree of evolution (stable, improving, getting worse), or an alert could be issued for the possibility of a heart attack in the next few hours.

\section{PROTOTYPE IMPLEMENTATION}

In order to validate the proposed framework, a system implementation was performed. Java is used as the programming language for the main modules. Fig. 3 shows the main dashboard for individual patient. Here, caregivers can monitor the patient health status data.

The first aspect that can be observed is the information about which patient is currently being viewed, and the option to switch to monitoring another patient. This makes it easy to monitor multiple patients on one device. Since this is a prototype implementation, only three types of sensors have been predefined, BPM (heart beats per minute), Body Temperature and Pressure. The last valid value received by the sensors will be displayed in the corresponding field. The simulated dashboard will receive data which would normally be sent directly by the IoT Data collection module; for example connected to a MySignals sensors. Although not using real sensors, the system will make the simulated data follow the same data flow within the processing module. The reported data aims to send generated linear sequence of data, so that specific situations can be tested, such as by example, a patient with a fever for many hours. Once received, this data may generate a notification to the health caregivers and the patient relatives. 


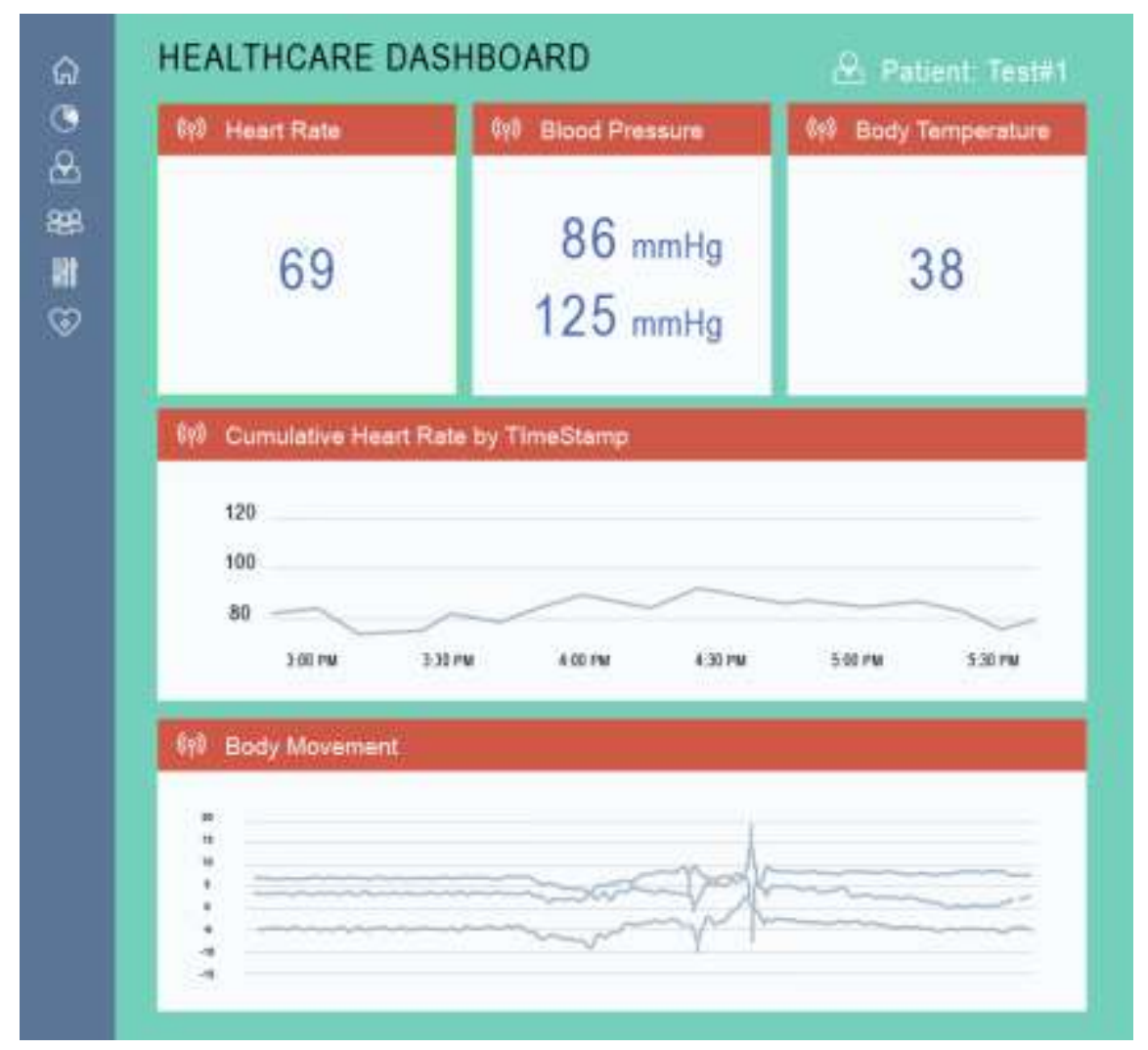

Fig. 3. Prototype Dashboard for the Collected Data.

\section{EXPERIMENTAL RESULTS}

In order to test the proposed Validation Module, specific rules were created to categorize patients infected with COVID19 into four profiles, based on disease severity.

To this end, the risk values were averaged, considering the lowest and highest values recorded in sensors of patients who recovered or not from COVID-19. Accordingly, the following profiles were created:

- Normal state (patients who have recovered).

- COVID aggravation stage 1.

- COVID aggravation stage 2 .

- COVID aggravation stage 3 .

After configuring the system and adding specific rules, the tests performed, using the module's simulated data linear sequence generation algorithm, it was possible to see that the simulated system correctly categorized the patients tested, according to the severity of the disease, indicated by the average values of the sensors.

Within a hospital environment there are different profiles of patients, with different diagnoses and being followed up in different ways. To reduce the scope of this work and simplify the evaluation of the results, the group of patients infected with COVID-19 and waiting (at home or in a hospital) for natural recovery or for the evolution of symptoms to proceed with hospitalization in a specific sector was chosen of the hospital environment.
The rules for generating predictions (which will be stored in the knowledge base) are not the direct target of this work, since this information would depend on the analysis of a specialist in the health area. Some rules are simple and even easily discovered, including in the manuals of health monitoring devices [24]. For example, the minimum glucose rate setting (which normally triggers an alert) could be set to 70 $\mathrm{mg} / \mathrm{dl}$ and the maximum normal temperature could be $36.7^{\circ} \mathrm{C}$. Other measures, however, need constant adjustments by health specialists.

\section{DISCUSSION}

In this paper, we proposed an IoT-based e-Health framework for COVID-19 patients monitoring, which is proved to be well-established as a useful and safe approach. Its use in for COVID-19 cases of risk allows adequate medical control, detects in advance the deteriorating of the disease, supports the care in times of high demand, helps to maintaining social isolation by avoiding calls to the emergency room and helps the patient and his relatives.

Although many previous works have been proposed in the control of acute infectious diseases, most of the existing evidence comes from the management of patients with chronic diseases. The evidence available on the role those new technologies such as IoT and advanced e-Health systems can play in controlling an epidemic is scarce.

For mitigating COVID-19, there are different studies (please see Section II) that provide guidance on the working method and the way to implement it. However, we believe that 
proposing a lightweight and effective framework for monitoring COVID-19 patients is beneficial. It is definitely a solid basis for future research, more extensive and with control groups, to define the role that IoT technologies have to play a major role in current and future pandemics.

The approach that combines IoT and proactive healthcare follow-up systems has been well accepted by the patients. Probably a main reason is that this monitoring strategy gives the user a security in days of uncertainty. The proactive attitude, the response to alerts or calls from patients in acceptable times gives a feeling of vigilance and control. We understand that, at least in part, this justifies that the connecting COVID-19 patients with health care givers is essential.

When assessing this work, a number of limitations must be considered. Firstly, the data used is simulated from small size of data and for a single hospital center as a use case. Since the findings may not be identical in areas with a different incidence of COVID-19, it is important to reproduce this study in other health areas.

In summary, this work suggests that IoT monitoring framework, used effectively, enabling useful and safe followup for high-risk COVID-19 patients, although steady at the time of diagnosis. More researches are required to validate these results and assess their possible application in potential pandemics.

\section{CONCLUSION}

New technologies for patient monitoring are very welcome and with the COVID-19 pandemic, they have become even more necessary. This work proposed a COVID-19 IoT-based framework that receives and analyzes patient data through a IoT sensors. An analysis of the proposed framework was also carried out, as well as implementing a prototype system.

As a result of this work, the proposed framework that works on an IoT-based sensor infrastructure is developed to provide real-time health information. The proposed framework is interconnectable with other systems, which process data and maintain a patient history. With the use of this framework, a general improvement in the quality of patient follow-up by the healthcare team is expected. This work was developed considering the monitoring exclusively of patients infected with COVID-19, but it is necessary to investigate whether it can be applicable to other diseases or health conditions.

\section{FUTURE WORK}

As for future work, it would be interesting to implement an application layer that can provide display data for medical caregivers, nurses and especially first responders. A possible hospital module could present information about each patient, including their current and past status, notification history and possible prognosis. A possible ambulance module could indicate this information and also all monitoring data in real time during the process of transferring the patient from home to the hospital, its alerts and prognoses. This could improve the efficiency of the entire process, as rescuers could anticipate events. Moreover, it would be essential to compare the efficiency of the proposed framework with relevant literature through the application of AI algorithms for detecting and monitoring COVID-10 cases.

\section{REFERENCES}

[1] M. Bansal, A. Goyal, and A. Choudhary, "Industrial Internet of Things (IIoT): A Vivid Perspective," Inventive Systems and Control, pp. 939949: Springer, 2021.

[2] M. Lombardi, F. Pascale, and D. Santaniello, "Internet of Things: A General Overview between Architectures, Protocols and Applications," Information, vol. 12, no. 2, pp. 87, 2021.

[3] G. Uganya, Radhika, and N. Vijayaraj, "A survey on internet of things: Applications, recent issues, attacks, and security mechanisms," Journal of Circuits, Systems Computers, vol. 30, no. 05, pp. 2130006, 2021.

[4] R. Ande, B. Adebisi, M. Hammoudeh, and J. Saleem, "Internet of Things: Evolution and technologies from a security perspective," Sustainable Cities Society, vol. 54, pp. 101728, 2020.

[5] A. Alamri, A. Gumaei, M. Al-Rakhami, M. M. Hassan, M. Alhussein, and G. Fortino, "An effective bio-signal-based driver behavior monitoring system using a generalized deep learning approach," IEEE Access, vol. 8, pp. 135037-135049, 2020.

[6] M. S. Al-Rakhami, A. Gumaei, M. Altaf, M. M. Hassan, B. F. Alkhamees, K. Muhammad, and G. Fortino, "FallDeF5: A Fall Detection Framework Using 5G-based Deep Gated Recurrent Unit Networks," IEEE Access, vol. 9, pp. 94299-94308, 2021.

[7] J. Delsing, J. Eliasson, J. van Deventer, H. Derhamy, and P. Varga, "Enabling IoT automation using local clouds." pp. 502-507, 2016.

[8] H. Magsi, A. H. Sodhro, N. Zahid, S. Pirbhulal, L. Wang, and M. S. AlRakhami, "A Novel Adaptive Battery-Aware Algorithm for Data Transmission in IoT-Based Healthcare Applications," Electronics, vol. 10, no. 4, pp. 367, 2021.

[9] P. Valsalan, T. A. B. Baomar, and A. H. O. Baabood, "IoT based health monitoring system," Journal of critical reviews, vol. 7, no. 4, pp. 739-743, 2020.

[10] Y. Shi, G. Wang, X.-p. Cai, J.-w. Deng, L. Zheng, H.-h. Zhu, M. Zheng, B. Yang, and Z. Chen, "An overview of COVID-19," Journal of Zhejiang University-SCIENCE B, vol. 21, no. 5, pp. 343-360, 2020.

[11] A. Gumaei, M. Al-Rakhami, M. M. Al Rahhal, F. R. H. Albogamy, E. Al Maghayreh, and H. AlSalman, "Prediction of COVID-19 confirmed cases using gradient boosting regression method," Computers, Materials Continua, vol. 66, no. 1, 2021.

[12] M. Otoom, N. Otoum, M. A. Alzubaidi, Y. Etoom, and R. Banihani, “An IoT-based framework for early identification and monitoring of COVID19 cases," Biomedical signal processing control, vol. 62, pp. 102149, 2020.

[13] X. Yang, X. Wang, X. Li, D. Gu, C. Liang, K. Li, G. Zhang, and J. Zhong, "Exploring emerging IoT technologies in smart health research: a knowledge graph analysis," BMC Medical Informatics Decision Making, vol. 20, no. 1, pp. 1-12, 2020.

[14] G. B. Rehm, S. H. Woo, X. L. Chen, B. T. Kuhn, I. Cortes-Puch, N. R. Anderson, J. Y. Adams, and C.-N. Chuah, "Leveraging IoTs and machine learning for patient diagnosis and ventilation management in the intensive care unit," IEEE Pervasive Computing, vol. 19, no. 3, pp. 68-78, 2020.

[15] O. Udawant, N. Thombare, D. Chauhan, A. Hadke, and D. Waghole, "Smart ambulance system using IoT." pp. 171-176, 2017.

[16] J. Lu, R. Jin, E. Song, M. Alrashoud, K. N. Al-Mutib, and M. S. AlRakhami, "An Explainable System for Diagnosis and Prognosis of COVID-19," IEEE Internet of Things Journal, 2020.

[17] R. P. Singh, M. Javaid, A. Haleem, and R. Suman, "Internet of things (IoT) applications to fight against COVID-19 pandemic," Diabetes Metabolic Syndrome: Clinical Research Review, vol. 14, no. 4, pp. 521$524,2020$.

[18] P. L. Penmatsa, and D. R. K. Reddy, "Smart detection and transmission of abnormalities in ecg via bluetooth." pp. 41-44, 2016.

[19] J. Van Zaen, O. Chételat, M. Lemay, E. M. Calvo, and R. DelgadoGonzalo, "Classification of cardiac arrhythmias from single lead ECG with a convolutional recurrent neural network," arXiv preprint arXiv:.01513, 2019. 
[20] C. Gutiérrez-Ardila, C. Montenegro-Marin, and P. Gaona-García, "IOT System for Self-diagnosis of Heart Diseases Using Mathematical Evaluation of Cardiac Dynamics Based on Probability Theory," Information Systems Technologies to Support Learning: Proceedings of EMENA-ISTL, vol. 111, pp. 433, 2018.

[21] M. A. Ponce-Gallegos, G. Pérez-Rubio, E. Ambrocio-Ortiz, N. PartidaZavala, R. Hernández-Zenteno, F. Flores-Trujillo, L. García-Gómez, A. Hernández-Pérez, A. Ramírez-Venegas, and R. Falfán-Valencia, "Genetic variants in IL17A and serum levels of IL-17A are associated with COPD related to tobacco smoking and biomass burning," Scientific reports, vol. 10, no. 1, pp. 1-11, 2020.
[22] H. Ahmadi, G. Arji, L. Shahmoradi, R. Safdari, M. Nilashi, and M. Alizadeh, "The application of internet of things in healthcare: a systematic literature review and classification," Universal Access in the Information Society, vol. 18, no. 4, pp. 837-869, 2019.

[23] X. Hu, A. M. Abdulghani, M. Imran, and Q. H. Abbasi, "Internet of Things (IoT) for Healthcare Application: Wearable Sleep Body Position Monitoring System Using IoT Platform." pp. 76-81, 2020.

[24] D. Boiroux, A. K. Duun-Henriksen, S. Schmidt, K. Nørgaard, S. Madsbad, N. K. Poulsen, H. Madsen, and J. B. Jørgensen, "Overnight glucose control in people with type 1 diabetes," Biomedical Signal Processing Control, vol. 39, pp. 503-512, 2018. 